\title{
Factor de amplificación dinámico ante la caída del carro de avance durante la construcción de un puente de viga cajón por el método de voladizos sucesivos Dynamic amplification factor due to sudden fall of the form traveler during the balanced cantilever construction of box girder bridges
}

\author{
Sergio Martínez-Leal ${ }^{1 \mathrm{a}}$, Daniela Osorio-Osorio ${ }^{1 \mathrm{~b}}$, José Benjumea-Royero ${ }^{1 \mathrm{c}}$, Homer Armando Buelvas-Moya ${ }^{1 \mathrm{~d}}$ \\ ${ }^{1}$ Escuela de Ingeniería Civil, Universidad Industrial de Santander, Bucaramanga, Colombia. Correos electrónicos: \\ a saml_93@hotmail.com, ${ }^{\mathrm{b}}$ daniela21osorio@outlook.es, ${ }^{\mathrm{c}}$ josbenro@uis.edu.co, ${ }^{\mathrm{d}}$ armando.buelvas@ hotmail.com
}

Recibido: 16 octubre, 2018. Aceptado: 21 febrero, 2019. Versión final: 25 mayo, 2019.

\begin{abstract}
Resumen
Dentro de las metodologías de construcción más utilizadas para puentes de viga cajón sobresale el método de voladizos sucesivos. En este método, la superestructura es construida de manera progresiva y cuasisimétrica respecto de las pilas usando dovelas hormigonadas in-situ. El proceso se basa en carros de avance apoyados en dovelas predecesoras que permiten la fundida de las siguientes dovelas. Ya que durante construcción existe el riesgo de la caída accidental del carro de avance, diferentes guías de diseño recomiendan estudiar esta situación mediante un análisis estático considerando la carga del carro en conjunto con un factor de amplificación dinámico (FAD) igual a 2.0. Debido a que este fenómeno es puramente dinámico y no estático, en este artículo se estudia la validez del enfoque pseudoestatico mediante el cálculo del FAD a partir de análisis dinámicos. En el estudio se investigaron la incidencia de la rigidez del tablero, la etapa constructiva y el tiempo y tipo de rotura sobre el FAD. Los resultados obtenidos demuestran que los valores más altos del FAD se presentan en tablero flexibles, y que la etapa constructiva critica corresponde a aquella antes de fundir la dovela de cierre. Además, se encontró que la recomendación de las guías de diseño es inadecuada, ya que es posible que ocurran FAD iguales a 3.0. Esto demuestra que el uso de análisis pseudoestáticos no es el correcto para esta situación accidental.
\end{abstract}

Palabras clave: puente viga cajón; voladizos sucesivos; caída del carro de avance; factores de amplificación dinámico.

\begin{abstract}
The balanced cantilever method stands out among the most commonly used construction methods for box girder bridges. In this method, the superstructure is built progressively and quasi-symmetrically with respect to the pier. Form travelers that are supported on a predecessor segment allow pouring the following one. The accidental fall of the form traveler is a potential risk during construction; thus, some design guidelines recommend the use of a dynamic amplification factor (DAF) of 2.0 to account for the dynamic effects during the event. The use of a static-analysis is performed along with the use of the DAF. The validity of this approach was investigated in this study by performing dynamic analysis on the girder flexural rigidity, on the construction stage at which the accident occurs and on the breakage time and function that simulate the accidental event. The larger values of DAF occurred in relatively flexible
\end{abstract}

ISSN impreso: 1657 - 4583, ISSN en línea: 2145 - 8456. CC BY-ND 4.0 (c) (i) $९$

S. Martínez-Leal, D. Osorio-Osorio, J. Benjumea-Royero, H. A. Buelvas-Moya, "Factor de amplificación dinámico ante la caída del carro de avance durante la construcción de un puente de viga cajón por el método de voladizos sucesivos," Rev. UIS Ing., vol. 18, no. 3, pp. 193-202, 2019. doi: 10.18273/revuin.v18n3-2019020 
superstructures, and the critical construction stages were those before the closure pour at mid-span. Moreover, the recommendation in the design guidelines was found inadequate as some of the analyzed cases had DAFs equal to 3.0. This demonstrates that the use pseudo-static analysis method is not the correct approach for these accidental situations.

Keywords: box girder bridge; balanced cantilevers; fall of the advance carriage; dynamic amplification factors.

\section{Introducción}

Los puentes de viga cajón se destacan como los más utilizados en la actualidad. Generalmente, estos puentes se construyen mediante el método de voladizos sucesivos usando carros de avance, lo cual resulta en una solución técnica y económicamente competitiva [1]. El diseño de estos puentes requiere un análisis riguroso mediante el cual se verifique la capacidad de los elementos para satisfacer condiciones de resistencia bajo diferentes situaciones durante construcción y en servicio [2]. Específicamente durante la construcción resulta de interés el estudio de los efectos dinámicos, debido a la caída accidental del carro de avance de construcción. Este fenómeno puede generarse por errores mecánicos en el carro de avance, lo que podría generar el colapso progresivo de los segmentos del puente en construcción [1].

Las guías de diseño, como el Eurocódigo [3], recomiendan el uso de un enfoque estático de carga accidental considerando el peso del carro en caída multiplicado por un factor de amplificación dinámica (FAD), que usualmente es igual a 2.0. Este método presenta varias debilidades revisadas en otras situaciones accidentales, como la rotura de cables en puentes atirantados [5-7, 9-11]. Por tanto, es de interés investigar su validez para el efecto de la caída accidental de carros de avance.

El estudio analítico fue realizado mediante modelos numéricos de un puente de luz total $220 \mathrm{~m}(60+100+60$ m) y sección viga cajón monocelular, el cual corresponde a un caso típico para puentes en viga cajón [9]. El FAD fue calculado a partir de los resultados de análisis dinámicos lineales aplicando las combinaciones de cargas de construcción recomendadas en AASHTO [2]. En el estudio se investigó además el efecto de la rigidez a flexión de la superestructura, la etapa constructiva en la que ocurre el evento accidental y el tiempo y tipo de rotura que simula el fenómeno.

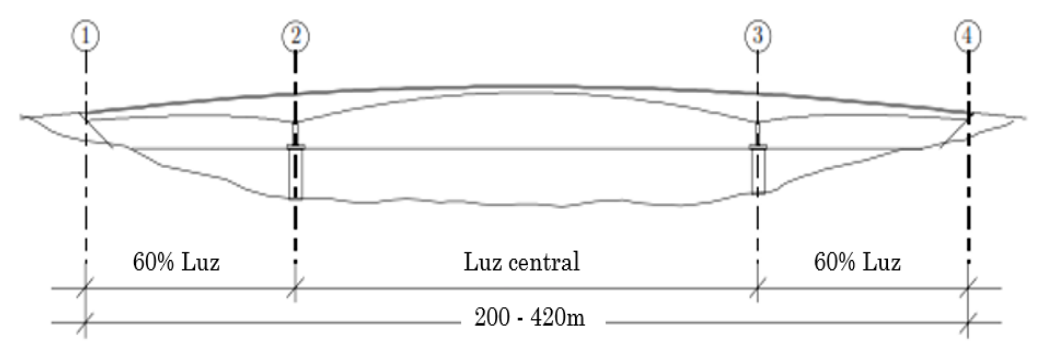

a)

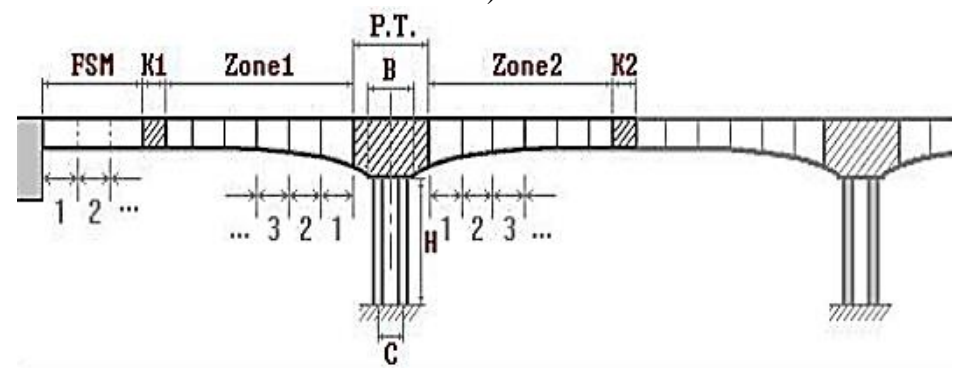

b)

Figura 1. Modelo típico de un puente viga cajón (a) y Secciones típicas del puente (b).

Fuente: Tomado de [2] (a) y elaboración propia (b). 


\section{Características del estudio}

\subsection{Descripción}

Los puentes estudiados tienen tres vanos, con una luz principal (L) de $100 \mathrm{~m}$ y dos vanos laterales iguales a $60 \mathrm{~m}$ $(0.6 \mathrm{~L})$.

Se asumieron conexiones rígidas en las uniones viga-pila y empotramientos en la base de las columnas (figura 1a). El tablero contiene una viga monocelular cuya altura fue modificada para investigar el uso de cantos constantes o variables. Para este último, la altura de la superestructura cambia desde $\mathrm{Hc}$ en el centro de luz a Hp en la pila. La relación $\mathrm{Hp} / \mathrm{Hc}$ se considera como parámetro variable, utilizando relaciones iguales a 1.0 (tipo M1), 0.5 (tipo M2) y 0.3 (tipo M3). Para el tablero de sección constante se usó una altura igual a $\mathrm{L} / 22$ y para secciones variables de $\mathrm{Hp}=\mathrm{L} / 16$ y en centros de luz $\mathrm{Hc}=\mathrm{L} / 55$ (figura 2).

Las dovelas de construcción tienen longitudes variables dependiendo de la zona de análisis. La zona de apoyo en la pila (Zona P.T. en figura 1) tiene dovelas de $3.825 \mathrm{~m}$ en el centro de apoyo y $3.75 \mathrm{~m}$ a lado y lado de dichas dovelas. Posterior a la zona P.T. se presenta la Zona 1 y la Zona 2 con dovelas de $3.75 \mathrm{~m}$ de longitud. En el centro de cada vano se encuentran las dovelas K: la dovela K1 en el centro del vano lateral de longitud $2.35 \mathrm{~m}$, y la K2 en el centro de la luz principal mide $2.175 \mathrm{~m}$. Finalmente, la zona de apoyo en los extremos (Zona F.S.M.) tiene dovelas de $3.0 \mathrm{~m}$ (Figura 1b).

En todos los casos estudiados el ancho total del tablero es $9.7 \mathrm{~m}$ con aletas de $2.15 \mathrm{~m}$ de longitud. De acuerdo con las recomendaciones en [2] se asumió un espesor de losa superior de $0.30 \mathrm{~m}$ y espesor de pared de los muros laterales del cajón de $0.45 \mathrm{~m}$. La losa inferior es variable en las secciones tipo M2 y tipo M3 (figura 2). Las alturas de Hc y Hp se resumen en la tabla 1.

Tabla 1. Dimensiones importantes de los modelos.

\begin{tabular}{|c|c|c|c|c|}
\hline $\begin{array}{c}\text { Relación } \\
\text { Hc/Hp }\end{array}$ & Modelo & $\begin{array}{c}\text { Recomendación } \\
\text { SETRA }\end{array}$ & $\begin{array}{c}\mathrm{Hp} \\
{[\mathrm{m}]}\end{array}$ & $\begin{array}{c}\mathrm{Hc} \\
{[\mathrm{m}]}\end{array}$ \\
\hline 1 & $\mathrm{M} 1$ & $\mathrm{~L} / 20$ & 4.55 & 4.55 \\
\hline 0.5 & $\mathrm{M} 2$ & $\mathrm{~L} / 16$ & 6.25 & 3.13 \\
\hline 0.3 & $\mathrm{M} 3$ & $\mathrm{~L} / 16$ & 6.25 & 1.88 \\
\hline
\end{tabular}

Fuente: elaboración propia.

\subsection{Modelamiento numérico}

Los puentes fueron analizados usando el software MIDAS CIVIL [4]. El tablero y las pilas se modelaron de acuerdo con las características del programa, mientras que los cables de postensionamiento fueron modelados como fuerzas y momentos aplicados en los extremos de las dovelas. Las propiedades de los materiales empleados en el modelamiento y diseño se presentan en la tabla 2.

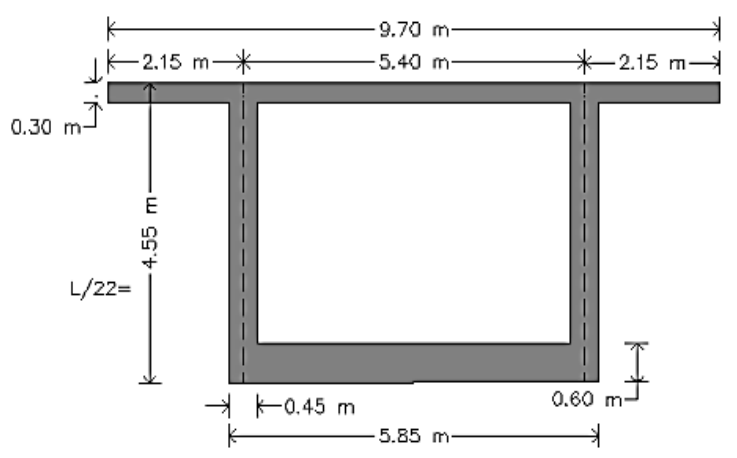

a)

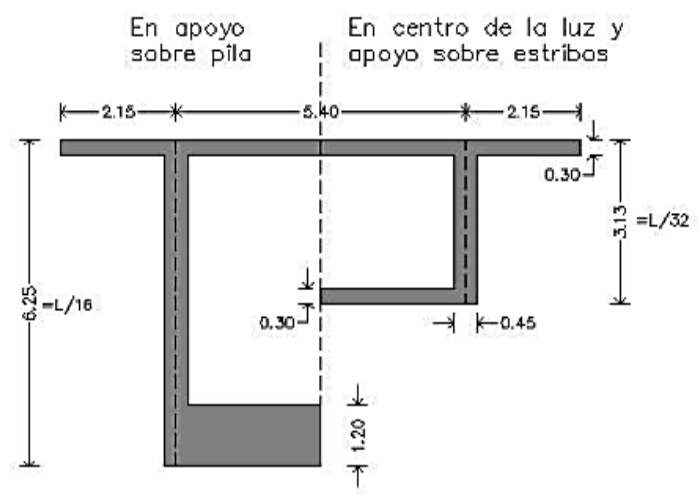

b)

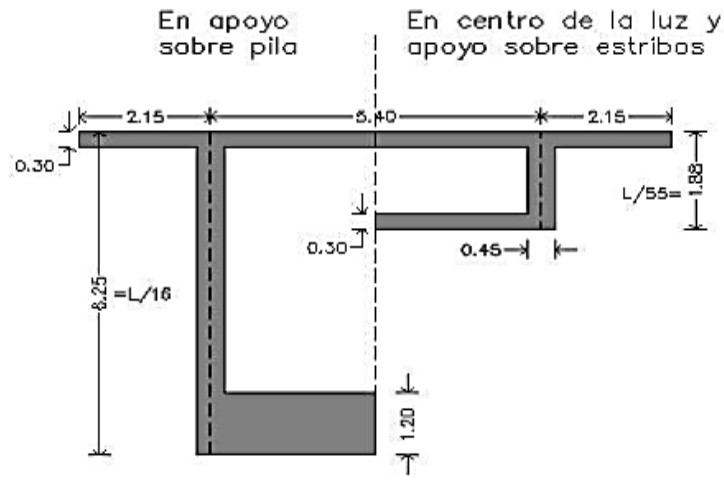

c)

Figura 2. Sección transversal puente viga cajón con sección constante $\mathrm{Hc} / \mathrm{Hp}=1$ (a) y sección variable $\mathrm{Hc} / \mathrm{Hp}=0.5$ (b) y $\mathrm{Hc} / \mathrm{Hp}=0.3$ (c).

Fuente: elaboración propia. 
Tabla 2. Datos de materiales en el modelo del puente.

\begin{tabular}{|l|c|c|}
\hline \multicolumn{1}{|c|}{ Característica } & Símbolo & Valor \\
\hline Resistencia a la compresión tablero & f'c & $41.36 \mathrm{MPa}$ \\
\hline Módulo de elasticidad tablero & $\mathrm{Ec}$ & $30772 \mathrm{MPa}$ \\
\hline Densidad especifica tablero & $\gamma$ & $24 \mathrm{kN} / \mathrm{m}^{3}$ \\
\hline Resistencia límite a compresión & $0.45 \mathrm{f}^{\prime} \mathrm{c}$ & $20.68 \mathrm{MPa}$ \\
\hline Resistencia límite a tracción & $0.25 \sqrt{ } \mathrm{f}$ 'c & $3.22 \mathrm{MPa}$ \\
\hline
\end{tabular}

Fuente: elaboración propia.

Se debe tener en cuenta que dentro del análisis paramétrico también se modificó progresivamente el lugar o etapa constructiva en la que ocurre la caída del carro de avance. Se trabajó con revisión en las etapas de construcción en L/4, L/2 y antes de la dovela de cierre. Las etapas constructivas (construction stage o CS) son la idealización simétrica del número de dovelas que se iban modelando de manera congruente con el lanzamiento de voladizos sucesivos con respecto a las pilas. La longitud de volados en una etapa inicial (CS2) tenía una dimensión de $11.325 \mathrm{~m}$ a lado y lado de la pila, para la segunda etapa (CS6) ya se contaba con 6 dovelas a lado y lado de la pila para una dimensión de 26.385 m; y para la etapa final (CS11) se validó el modelo para una longitud de volado de $44.053 \mathrm{~m}$ (figura 3).

Las cargas usadas en el análisis y diseño de los puentes corresponden a las definidas en AASHTO [1]. En todos los casos se verificó que los puentes prototipos satisfagan los estados de construcción (Construction Limit State, CLS) presentados en la tabla 3.

En dicha tabla, DC representa la carga muerta y de peso propio, DIFF es la carga diferencial en el voladizo central ( $2 \%$ peso propio), CLL es la carga viva de construcción $(5 \mathrm{kN} / \mathrm{m}$ en la luz central y $2.5 \mathrm{kN} / \mathrm{m}$ en los vanos laterales), CEQ es el peso del carro de avance $(600 \mathrm{kN})$ y WUP es la carga vertical debido al viento $(2.5 \mathrm{kN} / \mathrm{m})$.
Tabla 3. Combinaciones de carga de construcción.

\begin{tabular}{|c|c|}
\hline Estado Límite & Combinación \\
\hline CLS a & DC+DIFF+CLL+CEQ \\
\hline CLS b & DC+ CLL+CEQ \\
\hline CLS c & DC+DIFF+0.7WUP+CEQ \\
\hline CLS d & DC+DIFF+CLL+WUP+CEQ \\
\hline
\end{tabular}

Fuente: elaboración propia.

\section{Cálculo de los FAD}

La caída del carro de avance se modeló asumiendo que los elementos permanecen en el rango lineal y que este cae en el vano lateral mientras en el vano central se funde una dovela in situ. La rigidez y resistencia de la estructura podrían afectarse durante dicho fenómeno; sin embargo, el análisis lineal fue considerado razonable debido a que el mismo comportamiento es implícitamente asumido en el análisis pseudoestatico recomendado en las guías de diseño. Con el fin de modelar la caída del carro mediante un evento dinámico, el peso de este se aplicó de manera instantánea usando las funciones paso y rampa mostradas en la figura 4. En estas se asume que la caída ocurre en un tiempo de rotura, como se puede observar en la figura 4. Estas funciones se aplicaron en el modelo de sección constante para la etapa constructiva CS2, ya que su resultado no depende de la etapa constructiva ni de la rigidez. Se asumió un amortiguamiento del $2 \%$ para el análisis.

La situación accidental de la caída del carro de avance es tenida en cuenta mediante la combinación de carga de construcción presente en la sección 5.14.2.3. $4^{\mathrm{a}}$ del código AASHTO [1] y presentada en la ecuación (1). a)

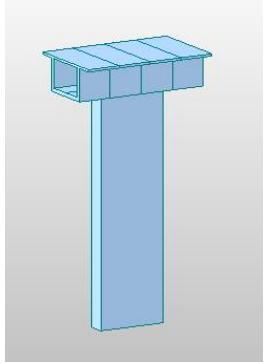

b)

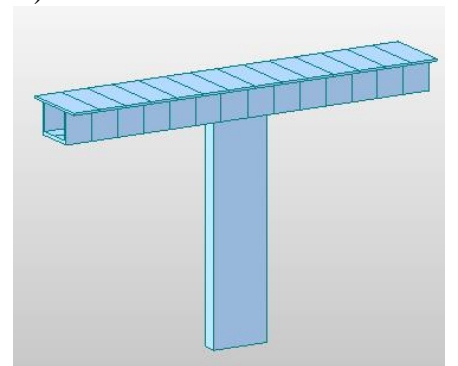

c)

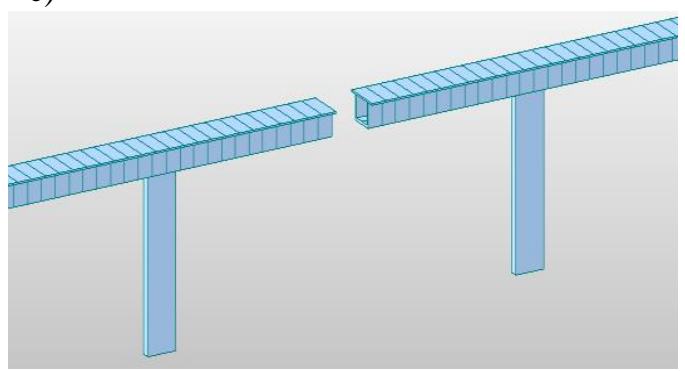

Figura 3. Etapa de construcción 1 CS2 (a), etapa de construcción 2 CS6 (b) y etapa final CS11 (c). Fuente: MIDAS 3D [4]. 


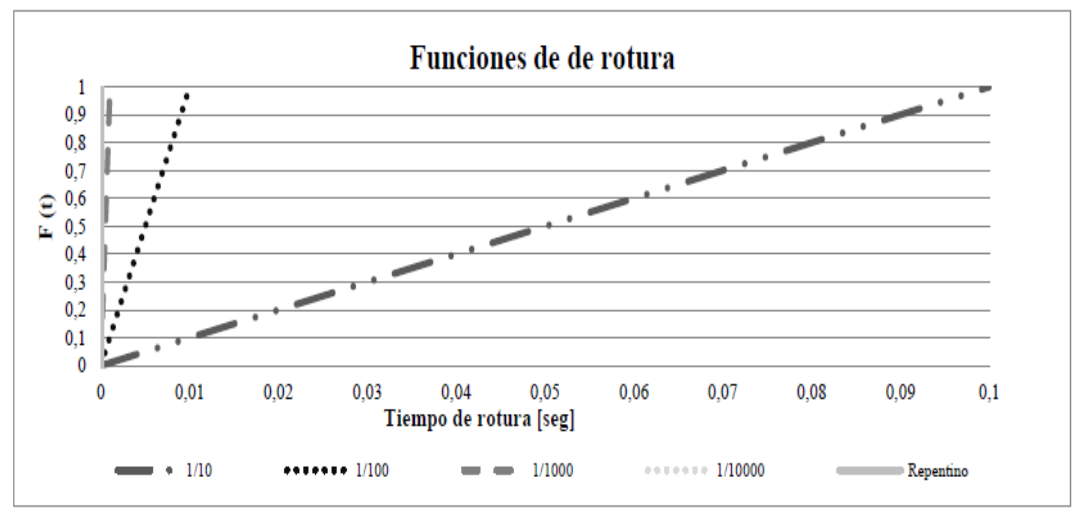

Figura 4. Funciones normalizadas de los modelos. Fuente: elaboración propia.

En esta ecuación de valores máximos y mínimos, $\mathrm{P}_{\mathrm{din}}$ corresponde a los efectos dinámicos generados por la rotura del cable:

$$
A L S=1.1(D C+D I F F)+1.3(C E Q+C L L)+P_{\text {din }}
$$

En general, el FAD representa una relación entre la respuesta de una estructura sometida a cargas dinámica y estática. En este trabajo se usaron dos expresiones para determinarlo.

En la primera Eq. 2, se analizó la respuesta del puente únicamente ante la carga de caída. En la segunda (Eq. 3), el puente es analizado bajo la acción de las cargas de construcción. Esto es, las cargas adicionales de la combinación presentada en la tabla 4. En ambas ecuaciones, $S_{\text {stat }}$ representa la respuesta del puente ante la aplicación de la carga estática, y $S_{d y n}$, la respuesta del puente ante la aplicación de la carga dinámica.

$$
\begin{gathered}
F A D(1)=\frac{S_{\text {dyn } \sin \text { cargas }}}{S_{\text {sta } \sin \text { cargas }}} \\
F A D(2)=\frac{S_{\text {dyn con cargas }}}{S_{\text {sta con cargas }}}
\end{gathered}
$$

En la unión de dovelas se presenta doble cálculo de FAD, y el análisis se realiza para los modelos M1, M2 y M3. Cabe resaltar que los modelos tienen un parámetro fijo relacionado con la ubicación de la caída del carro de avance. Como referencia del modelo, se tomó la carga accidental a lado izquierdo del puente, como se aprecia en la figura 5. Según [9], la carga accidental se idealiza contraria a la carga peso del carro de avance y se pone en dicho sector de aplicación, según corresponda en la etapa de construcción CS2, CS6 y CS11.

\section{Resultados}

Para idealizar los resultados, se realizó el cálculo del FAD como se describió anteriormente y a lo largo del lado izquierdo del tablero. Se describen FAD máximos y mínimos. Se seleccionó la función repentina porque presentaba los mayores efectos en el cálculo del FAD, tal como se puede analizar en la figura 6 .

Los valores de la función repentina generan mayores efectos en el comportamiento dinámico y, por tanto, el numerador de las Eq. 2 y Eq. 3, relacionados directamente con el valor de FAD.
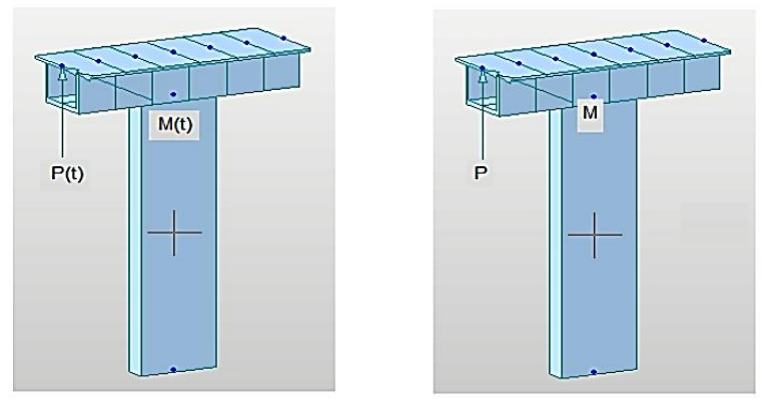

Figura 5. Modelos de aplicación de carga a lado izquierdo de la pila.

Fuente: elaboración propia.

\subsection{Resultados M1}

Se realiza la evaluación del FAD a lo largo del tablero para las etapas CS2, CS6 y CS11, se inicia con los modelos M1 (o de sección transversal contante), tal como se observa en la figura 7.

Realizando un análisis de la etapa CS2 (fig. 7a.) solo se obtienen valores particulares en cercanía a la pila con valor de 2.7 aproximadamente. Para la etapa CS6 (Fig. $7 b$.) no se supera el FAD igual a 2.0 en ningún caso, y la 


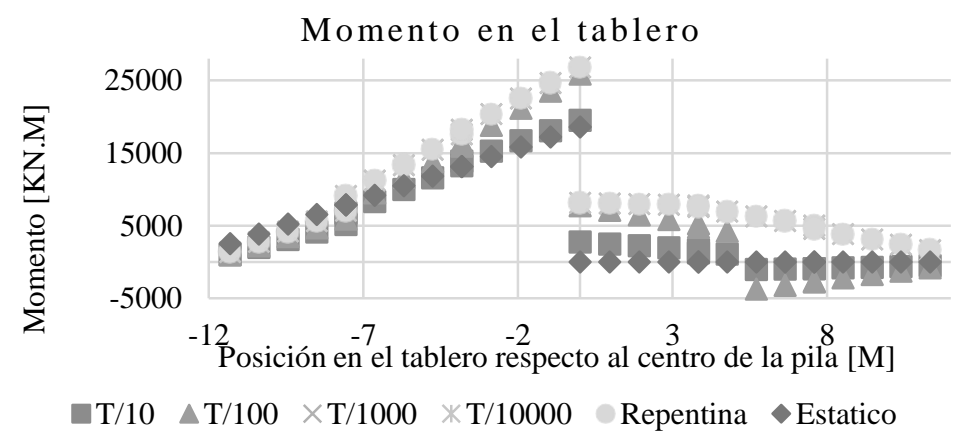

Figura 6. Funciones normalizadas de los modelos. Fuente: elaboración propia.

tendencia es a aumentar sus valores en secciones cercanas a la pila.

La gráfica de comportamiento de la etapa CS11 (fig. 7c.) representa valores de FAD inferiores a 2 en la mayor del puente, pero con tendencia asíntota a infinito en cercanías a la pila y al lugar del caído de carro de avance. Lo que indica que la respuesta estática era muy pequeña con respecto a la respuesta dinámica del puente.

\subsection{Resultados M2}

Se realiza la evaluación del FAD a lo largo del tablero para las etapas CS2, CS6 y CS11, los resultados del modelo $\mathrm{Hc} / \mathrm{Hp}=0.5$ se muestran en la figura 8 .

Realizando un análisis de la etapa CS2 (Fig. 8a.), se obtienen valores particulares en cercanía al lugar de caída del carro de avance (4 metro). Se presenta una asíntota de datos en cercanías al carro de avance, indicando que la respuesta estática es casi nula en esos sectores (denominador de las Eq.2 y Eq. 3 tiende a cero, FAD tiende a infinito).

Para la etapa CS6 (fig. 8b.), el valor de FAD supera el dato de 2.0, siendo la tendencia a aumentar sus valores en secciones cercanas a la pila, detallándose FAD similares a 6.0. La gráfica de comportamiento de la etapa CS11 (Fig. 8c.) representa valores de FAD inferiores a 2 en la mayor del puente, pero con tendencia asíntota a infinito en cercanías a la pila y en el lugar de la caída del carro de avance. Lo que indica, al igual que para M1, que la respuesta estática era muy pequeña con respecto a la respuesta dinámica del puente.

\subsection{Resultados M3}

Se realiza la evaluación del FAD a lo largo del tablero para las etapas CS2, CS6 y CS11, los resultados del modelo $\mathrm{Hc} / \mathrm{Hp}=0.3$ se muestran en la figura 10 .
Desarrollando un análisis de la etapa CS2 (fig. 10a.), se obtiene un comportamiento similar a M2. Se presenta una asíntota de datos en cercanías al lugar de caída del carro de avance, lo que indica que la respuesta estática es casi nula en esos sectores.

Para la etapa CS6 (fig. 10b.), el valor de FAD supera el dato de 2.0, y la tendencia es a aumentar sus valores en secciones cercanas a la pila; se detallan FAD similares a 8.0 o superiores. La gráfica de comportamiento de la etapa CS11 (fig. 10c.) representa valores de FAD en promedio superiores a 2 en la mayor parte del tablero, pero con tendencia asíntota a infinito en cercanías a la pila y al lugar de la caída del carro de avance. Lo que indica, al igual que para M1y M2, que la respuesta estática era muy pequeña con respecto a la respuesta dinámica del puente, pero que la influencia de la respuesta dinámica es mayor al generar valores apreciables del FAD.

\section{Conclusiones}

Los resultados del estudio permiten concluir:

- La función de rotura crítica corresponde a la propia que idealiza la caída súbita del carro de avance.

- $\mathrm{El} \mathrm{FAD} \mathrm{=} 2.0$ recomendado en normas y guías de diseño fue superado en la mayoría de los casos estudiados. Su aplicabilidad no es pertinente para todos los componentes del puente.

- Ignorando los efectos de indeterminacion de las Eq.2 y Eq.3., el mayor factor de amplificación FAD se presentó en la dovela pila del modelo M1 $(\mathrm{hc} / \mathrm{hp}=1)$ con un valor superior 2.7. Pero los mayores efectos se presentaron en la etapa anterior a la construcción de la dovela cierre.

- A menor rigidez de la superestructura, mayor FAD (asumiendo pila constante). 
FAD Momento tablero M1 etapa CS2

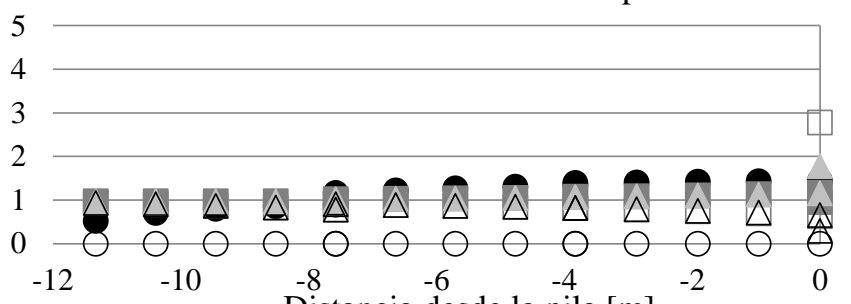

Distancia desde la pila [m]

-F.A.D ecuación (1) Max

OF.A.D ecuación (1) Min

F.A.D ecuación (2) fuerzas máximas Max

$\square$ F.A.D ecuación (2) fuerzas máximas Min

$\Delta$ F.A.D ecuación (2) fuerzas mínimas Max

a)

FAD Momento tablero M1 etapa CS6

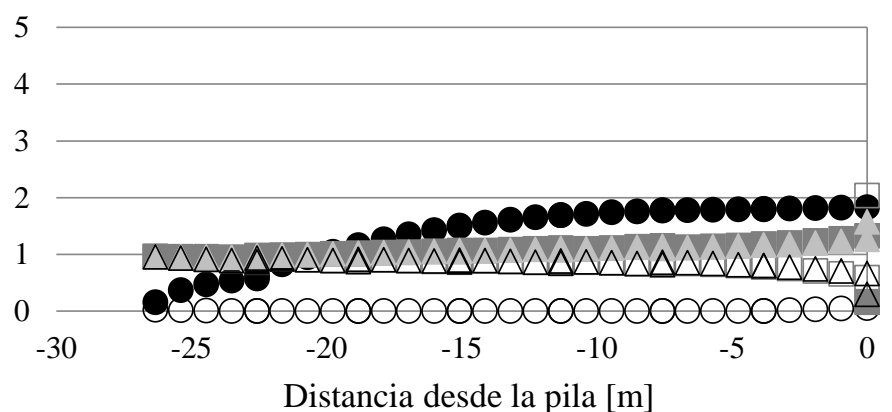

-F.A.D ecuación (1) Max

OF.A.D ecuación (1) Min

F.A.D ecuación (2) fuerzas máximas Max

$\square$ F.A.D ecuación (2) fuerzas máximas Min

$\triangle$ F.A.D ecuación (2) fuerzas mínimas Max

$\triangle$ F.A.D ecuación (2) fuerzas mínimas Min

b)

FAD Momento tablero M1 etapa CS11

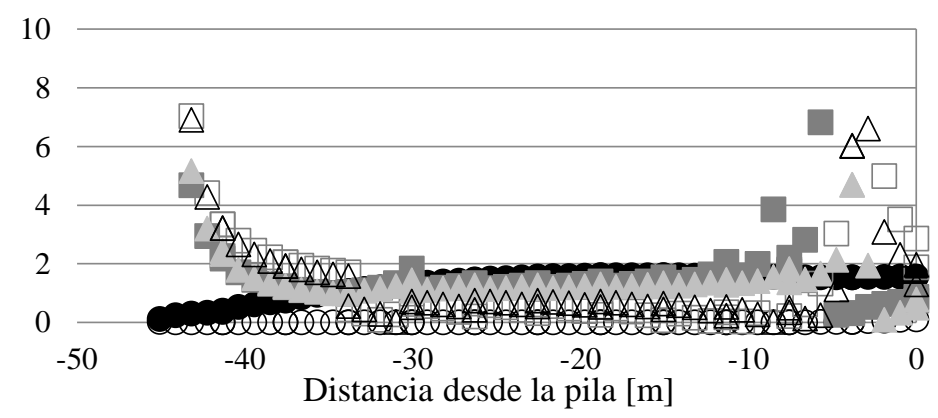

-F.A.D ecuación (1) Max

OF.A.D ecuación (1) Min

F.A.D ecuación (2) fuerzas máximas Max

$\square$ F.A.D ecuación (2) fuerzas máximas Min

$\triangle$ F.A.D ecuación (2) fuerzas mínimas Max

c)

Figura 7. Resultados FAD en la etapa CS2 (a), CS6 (b) y CS11 (c) para el modelo M1 (Hc/Hp=1).

Fuente: elaboración propia. 
FAD Momento tablero M2 etapa CS2

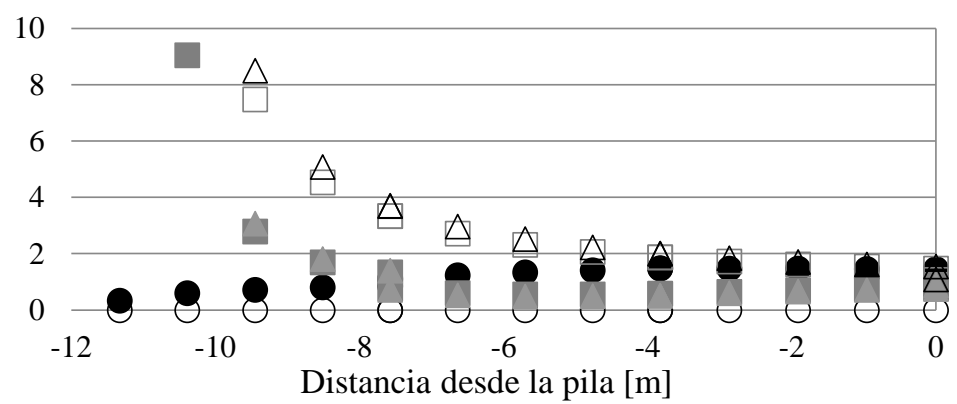

- F.A.D ecuación (1) Max

OF.A.D ecuación (1) Min

F.A.D ecuación (2) fuerzas máximas Max

$\square$ F.A.D ecuación (2) fuerzas máximas Min

$\Delta$ F.A.D ecuación (2) fuerzas mínimas Max

a)

FAD Momento tablero M2 etapa CS6

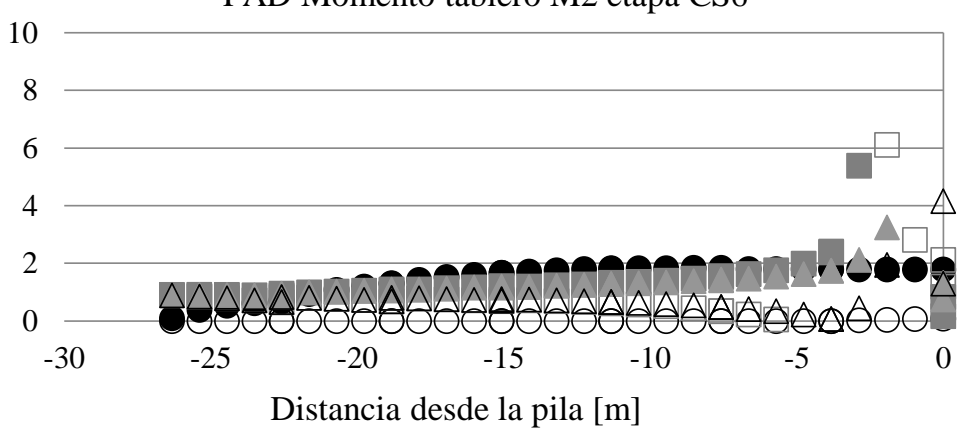

-F.A.D ecuación (1) Max

OF.A.D ecuación (1) Min

F.A.D ecuación (2) fuerzas máximas Max

$\square$ F.A.D ecuación (2) fuerzas máximas Min

$\Delta$ F.A.D ecuación (2) fuerzas mínimas Max

b)

FAD Momento tablero M2 etapa CS11

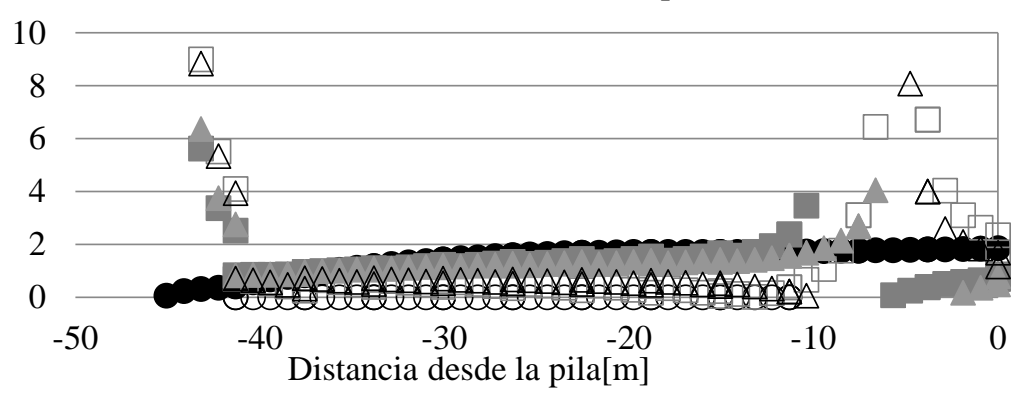

F.A.D ecuación (1) Max

OF.A.D ecuación (1) Min

F.A.D ecuación (2) fuerzas máximas Max

$\square$ F.A.D ecuación (2) fuerzas máximas Min

c)

Figura 8. Resultados FAD en la etapa CS2 (a), CS6 (b) y CS11 (c) para el modelo M2 (Hc/Hp=0.5).

Fuente: elaboración propia. 
FAD Momento tablero M3 etapa CS2

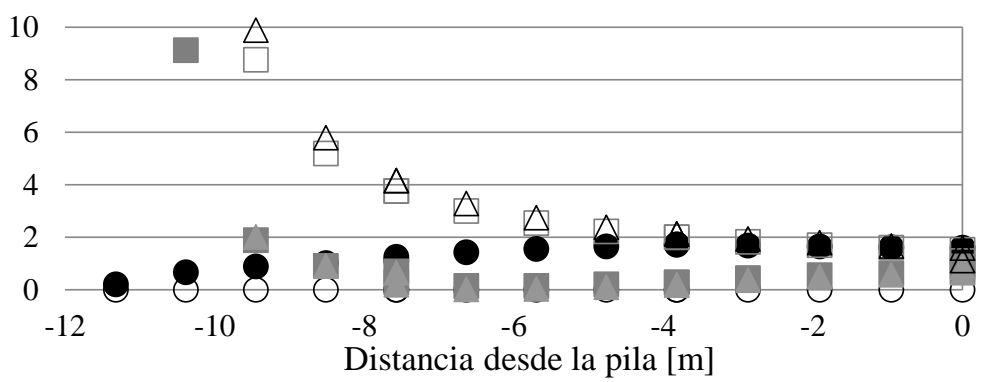

-F.A.D ecuación (1) Max

OF.A.D ecuación (1) Min

F.A.D ecuación (2) fuerzas máximas Max

$\square$ F.A.D ecuación (2) fuerzas máximas Min

$\triangle$ F.A.D ecuación (2) fuerzas mínimas Max

a)

FAD Momento tablero M3 etapa CS6

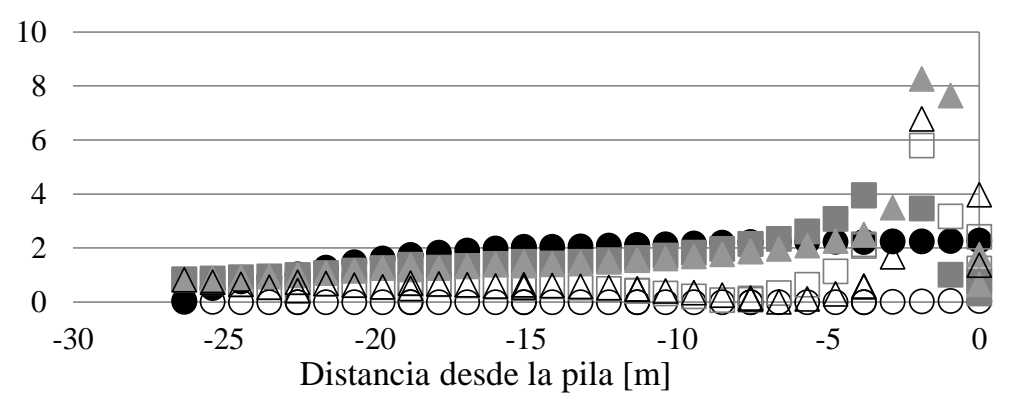

-F.A.D ecuación (1) Max

OF.A.D ecuación (1) Min

F.A.D ecuación (2) fuerzas máximas Max

$\square$ F.A.D ecuación (2) fuerzas máximas Min

$\Delta$ F.A.D ecuación (2) fuerzas mínimas Max

b)

FAD Momento tablero M3 etapa CS11

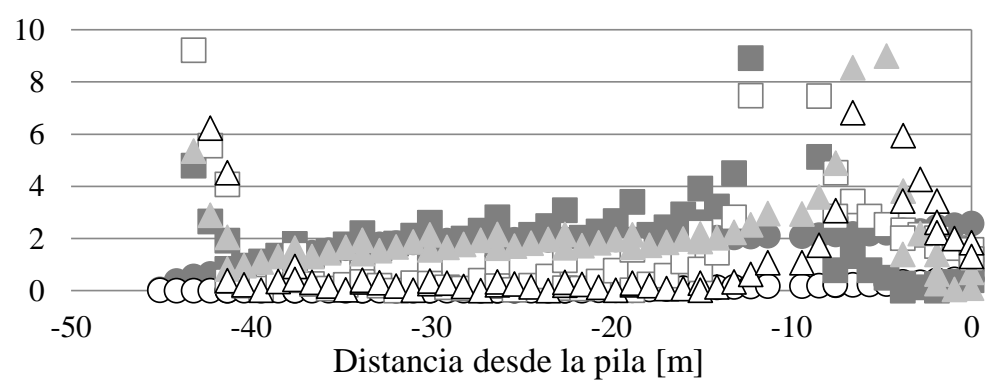

-F.A.D ecuación (1) Max

OF.A.D ecuación (1) Min

F.A.D ecuación (2) fuerzas máximas Max

$\square$ F.A.D ecuación (2) fuerzas máximas Min

$\triangle$ F.A.D ecuación (2) fuerzas mínimas Max

c)

Figura 10. Resultados FAD en la etapa CS2 (a), CS6 (b) y CS11 (c) para el modelo M3 (Hc/Hp=0.3).

Fuente: elaboración propia. 


\section{Referencias}

[1] P. F. Takacs, "Deformations in concrete cantilever bridges: Observations and theoretical modelling.," The Norwegian University of Science and Technology, 2004.

[2] AAHSTO Código Americano de Diseño Sísmico de Puentes, AAHSTO, 2012.

[3] K. Ahmadi-Kashani, "The landmark Metsovitikos Bridge, Greece," Proc. Inst. Civ. Eng. - Bridg. Eng., vol. 161, no. 4, pp. 165-176, 2008, doi: 10.1680/bren.2008.161.4.165.

[4] The European Union, Eurocode 1: Actions on structures - Part 1-7: General actions - Accidental actions. 2006.

[5] MIDAS CIVIL 3D. Licencia Universidad Industrial de Santander.

[6] C. M. Mozos and A. C. Aparicio, "Parametric study on the dynamic response of cable stayed bridges to the sudden failure of a stay, Part I: Bending moment acting on the deck," Eng. Struct., vol. 32, no. 10, pp. 32883300, 2010, doi: 10.1016/j.engstruct.2010.07.003.

[7] C. M. Mozos and A. C. Aparicio, "Parametric study on the dynamic response of cable stayed bridges to the sudden failure of a stay, Part II: Bending moment acting on the pylons and stress on the stays," Eng. Struct., vol. 32, no. 10, pp. 3301-3312, 2010, doi: 10.1016/j.engstruct.2010.07.002.

[8] C. M. Mozos and A. C. Aparicio, "Numerical and experimental study on the interaction cable structure during the failure of a stay in a cable stayed bridge," Eng. Struct., vol. 33, no. 8, pp. 2330-2341, 2011, doi: 10.1016/j.engstruct.2011.04.006.

[9] A. M. Ruiz-Teran and A. C. Aparicio, "Dynamic amplification factors in cable-stayed structures," $J$. Sound Vib., vol. 300, no. 1, pp. 197-216, 2007, doi: 10.1016/j.jsv.2006.07.028.

[10] A. Ruiz-Teran and A. Aparicio, eliminating bridge piers using stay cables with unconventional layouts. Taylor \& Francis Group, 2008. doi: 10.1201/9781439828410.ch105

[11] A. M. Ruiz-Teran and A. C. Aparicio, "Response of under-deck cable-stayed bridges to the accidental breakage of stay cables," Eng. Struct., vol. 31, no. 7, pp. 1425-1434, 2009, doi: 10.1016/j.engstruct.2009.02.027.
[12] M. Rosignoli, "Robustness and Stability of Launching Gantries and Movable Shuttering Systems Lessons Learned," Struct. Eng. Int., vol. 17, no. 2, pp. 133-140, May 2007, doi: $10.2749 / 101686607780680673$.

[13] Setra, Haubans. "Recommandations de la Commission Interministerielle de la Precontrainte". Service d'Etudes Techniques des Routes et Autoroutes, Bagneux, Francia, 2001.

[14] W.-F. Chen and L. Duan, Handbook of international bridge engineering. Taylor and Francis, 2013.

[15] M. Wolff and U. Starossek, "Cable loss and progressive collapse in cable-stayed bridges," Bridg. Struct., vol. 5, no. 1, pp. 17-28, Mar. 2009, doi: $10.1080 / 15732480902775615$. 